\title{
Nasopharyngeal carcinoma with orbital invasion
}

W-M Hsu and A-G Wang

\section{Introduction}

Nasopharyngeal carcinoma (NPC) has its own distinct epidemiological pattern. It occurs much more frequently in the Chinese and other south-east Asians, and is most prevalent in the areas of south China, Hong-Kong, Taiwan, and south-east Asia. ${ }^{1}$ In mixed racial countries such as Singapore, the Chinese have a much higher rate of NPC than Malays and Indians. ${ }^{1}$ In addition, NPC in different races may have different manifestations, such as different age distribution of the tumour. ${ }^{1}$ Nevertheless, people in northern China have a relatively low incidence of the NPC. This distinct pattern indicates that both genetic and environmental factors are important in the pathogenesis of NPC.

In Taiwan, neck mass was the most common presenting symptom of NPC patients. ${ }^{2}$ Bloodtinged sputum and/or rhinorrhoea was the second most frequent symptom. ${ }^{2}$ Nasal symptoms (nasal obstruction, discharge), aural symptoms (tinnitus, fullness, hearing loss), headache, and cranial neuropathy were also noted. Cranial nerve involvement was observed in $22 \%$ of NPC patients at the first examination. ${ }^{2}$ Cranial neuropathy occurs when the skull base is invaded by the tumour.

NPC usually arises from the lateral wall of the nasopharynx, especially around the fossa of Rosenmuller and the Eustachian cushion. NPC has a great propensity to spread and infiltrate into adjacent tissues. It may spread superiorly to involve the skull base and intracranium, resulting in cranial nerve involvement. ${ }^{1}$ The incidence of skull base and brain invasion has been reported in some studies to be $12-31 \%$ in NPC patients. ${ }^{3-5}$ It may also invade anteriorly to the nasal cavity, paranasal sinuses, pterygopalatine fossa, and apex of orbit. ${ }^{1}$ Direct invasion of the orbit, however, is relatively rare in NPC patients. In this series, we report 13 cases of NPC with orbital invasion, describe the clinical
Department of

Ophthalmology

Taipei Veterans General Hospital School of Medicine National Yang-Ming University

Taipei, Taiwan

Correspondence:

W-M Hsu

Department of

Ophthalmology

Taipei Veterans General

Hospital

Taipei 11217, Taiwan

Tel: + 886228757325

Fax: + 886228761351

E-mail:wmhsu@vghtpe. gov.tw

Received: 16 September 2003

Accepted in revised form:

23 October 2003

Published online:

27 February 2004

Part of this work has been presented as a poster at the annual meeting of American Academy of Ophthalmology, 1997, San Francisco, USA
Keywords: invasion; nasopharyngeal carcinoma; orbit; orbital tumor 
Table 1 Clinical data of NPC with orbital invasion

\begin{tabular}{|c|c|c|c|c|c|c|c|c|c|}
\hline Case no. & Sex & Age & Laterality & Initial vision & Final vision & Previous NPC & Treatment $^{a}$ & Follow-up (month) & Outcome \\
\hline 1 & M & 64 & $\mathrm{R}$ & $6 / 10$ & $6 / 15$ & Y & 1 & 24 & Alive \\
\hline 2 & M & 54 & $\mathrm{R}$ & $6 / 7.5$ & $6 / 10$ & $\mathrm{Y}$ & $1+3$ & 28 & Alive \\
\hline 3 & M & 32 & $\mathrm{~L}$ & $6 / 10$ & $6 / 10$ & $\mathrm{~N}$ & $1+3$ & 40 & Alive \\
\hline 4 & M & 68 & $\mathrm{~L}$ & $6 / 60$ & $\mathrm{CF}$ & Y & $1+3$ & 4 & Dead \\
\hline 5 & $\mathrm{~F}$ & 54 & $\mathrm{~L}$ & $6 / 10$ & ND & $\mathrm{Y}$ & 5 & 4 & Lost \\
\hline 6 & M & 47 & $\mathrm{R}$ & $6 / 12$ & $6 / 10$ & Y & $1+2$ & 64 & Dead \\
\hline 7 & M & 57 & $\mathrm{~L}$ & $6 / 7.5$ & $6 / 15$ & $\mathrm{~N}$ & $1+3$ & 28 & Alive \\
\hline 8 & M & 56 & $\mathrm{R}$ & NLP & NLP & $\mathrm{Y}$ & $1+4$ & 18 & Alive \\
\hline 9 & M & 68 & $\mathrm{~L}$ & $3 / 60$ & $\mathrm{CF}$ & $\mathrm{N}$ & 1 & 6 & Dead \\
\hline 10 & M & 54 & $\mathrm{~L}$ & $6 / 30$ & $6 / 20$ & Y & $1+2$ & 28 & Dead \\
\hline 11 & M & 51 & $\mathrm{R}$ & $6 / 7.5$ & $6 / 12$ & $\mathrm{~N}$ & $1+2$ & 24 & Alive \\
\hline 12 & M & 70 & L & $6 / 20$ & $6 / 60$ & $\mathrm{Y}$ & $1+3$ & 8 & Dead \\
\hline 13 & $\mathrm{~F}$ & 43 & $\mathrm{~L}$ & $6 / 6$ & NLP & $\mathrm{Y}$ & $1+2$ & 52 & Dead \\
\hline
\end{tabular}

ND: not determined, NLP: no light perception, CF: finger counting.

${ }^{a}$ Treatment modalities: $1=$ radiotherapy, $2=$ chemotherapy, $3=$ orbitotomy, $4=$ enucleation, $5=$ none.

findings, and discuss the incidence, symptoms and signs, treatment, and prognosis in these patients.

\section{Materials and methods}

Setting: Taipei Veterans General Hospital and Taichung Veterans General Hospital.

Patients: We searched the medical records and computer registry of the Department of Ophthalmology at Taipei Veterans General Hospital and Taichung Veterans General Hospital, and then studied the cases of 13 patients diagnosed with NPC with orbital invasion between January 1974 and December 2001. Those patients without definite pathologic diagnosis were excluded. The pathologic diagnoses were made from orbital and/or nasopharyngeal biopsies (incisional or needle biopsy). Patients without ophthalmic examination were also excluded.

Observational procedures: We reviewed the chart records and imaging studies of these patients. Chart records included demographic information, ophthalmic examinations, treatment modalities, and clinical outcomes. Imaging studies included computed tomography (CT) and magnetic resonance images (MRI).

Outcome measures: The data were analysed and the clinical courses of four cases were studied in further detail. The findings in these four cases are discussed at length in the 'Results' section. Survival analysis was carried out using a Kaplan-Meier estimate.

\section{Results}

A total of 13 patients (11 males, two females) were included in this study. The ages of the patients ranged from 32 to 70 years old, with a mean age of 55 years.
Table 2 Ocular manifestation of orbital NPC $(n=13)$

\begin{tabular}{lc}
\hline Manifestation & Cases (\%) \\
\hline Proptosis & $12(92.3 \%)$ \\
Diplopia & $9(69.2 \%)$ \\
Blurred vision & $8(61.5 \%)$ \\
Orbital pain & $6(46.1 \%)$ \\
Opti disc Oedema & $2(15.3 \%)$ \\
Lid mass & $1(7.6 \%)$ \\
Optic atrophy & $2(15.3 \%)$ \\
\hline
\end{tabular}

Right orbit invasion was noted in five cases, while left orbit invasion existed in eight cases (Table 1). Proptosis was the most common symptom in the NPC patients with orbital invasion. Diplopia, blurred vision, and orbital pain were frequently found as well (Table 2). Among the 13 patients, nine had been previously diagnosed with NPC, and the remaining four cases presented ocular and/or orbital symptoms as the initial manifestation of NPC. Of these four patients, all were males and aged from 32 to 68 years. Interestingly, one patient presented with eyelid tumour (Case 9) and three patients presented with proptosis (Cases $3,7,11$ ) as the first manifestations of NPC. When three of the four patients presented, their vision was better than $6 / 10$. The 32 -year-old patient was the youngest patient in this study and he was followed for 34 months with a vision of $6 / 10$.

The treatment modalities of the patients were varied (Table 1). In total, 12 patients received radiotherapy (7000 rad) for NPC, but one patient (Case 5) refused any further treatment and was subsequently lost during follow-up. Among the 12 patients receiving radiotherapy, two patients received radiotherapy alone and the other 10 patients received radiotherapy combined with other treatment modalities. Five patients received orbitotomy 
to remove orbital tumour (partial or total), four received radiotherapy combined with chemotherapy, and one received enucleation due to painful useless eye.

During the follow-up period of 4-64 months, the outcome analysis (Table 1) revealed that six patients died. As stated previously, Case 5 had been lost to follow-up, but the remaining six patients were still alive. The 5-year survival rate in these patients was $28 \%$ (Figure 1).

\section{Case reports}

\section{Case 6}

This 47-year-old male patient complained of nasal obstruction and paraesthesia over right lip area for about 2 months. He suffered from gradual impairment of vision in the right eye for 1 month. Physical examination did not reveal any sign of neck lymphadenopathy. Neurologic examination found right facial paresis and paraesthesia. Ophthalmic examination revealed poor vision in the right eye and his visual acuity was determined to be finger counting in this eye. Mild limitation of extraocular movement in all directions was

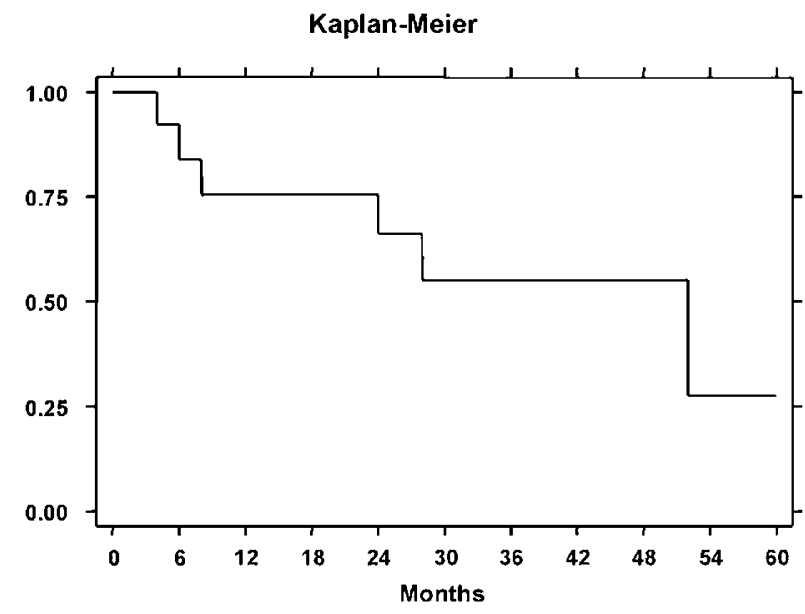

Figure 1 Survival analysis of all 13 patients was carried out with a Kaplan-Meier survival estimate. The 5-year survival rate was determined to be $28 \%$. also found in the right eye, in which the anterior segment was normal, except for the Marcus-Gunn pupil. Fundus examination was normal in both eyes. Visual field revealed a central scotoma in the right eye.

Computerized tomography showed an ill-defined mass over the nasopharynx and right orbital apex region. The biopsy report showed epidermoid carcinoma of the nasopharynx. The patient received chemotherapy and radiotherapy. After 4 years, the patient was rather well with the exception of optic atrophy in the right eye with a vision of finger counting. Unfortunately, the patient died as a result of an unrelated accident in the 64th month of the follow-up period.

\section{Case 7}

A 57-year-old male patient presented with left eye proptosis for 6 months. His past history was unremarkable. Ophthalmic examination revealed mild proptosis in the left eye. Extraocular movement was full in both eyes. His vision was 6/7.5 in the left eye. Slitlamp examination showed a mild degree of nuclear sclerosis in both lenses. Fundus examination was normal in both eyes. A CT scan of the orbit showed a mass at the inferior orbit and maxillary sinus. Orbitotomy was then performed to remove the tumour and the pathological report proved his condition to be NPC. He received radiotherapy and was followed for 28 months with a final vision of $6 / 15$.

\section{Case 9}

A 68-year-old male presented with a huge left eyelid mass for more than one year. Vision was $6 / 120$ in the right eye and finger counting in the left eye. Ophthalmic examination revealed a protruding mass, about $3.7 \mathrm{~cm}$ in diameter, at the medial canthal region (Figure $2 \mathrm{a}$ ). The surface of the tumour was irregular, with ulceration and whitish discharge. Orbital CT scan showed a large mass occupying the orbital and nasal cavities, which seems to
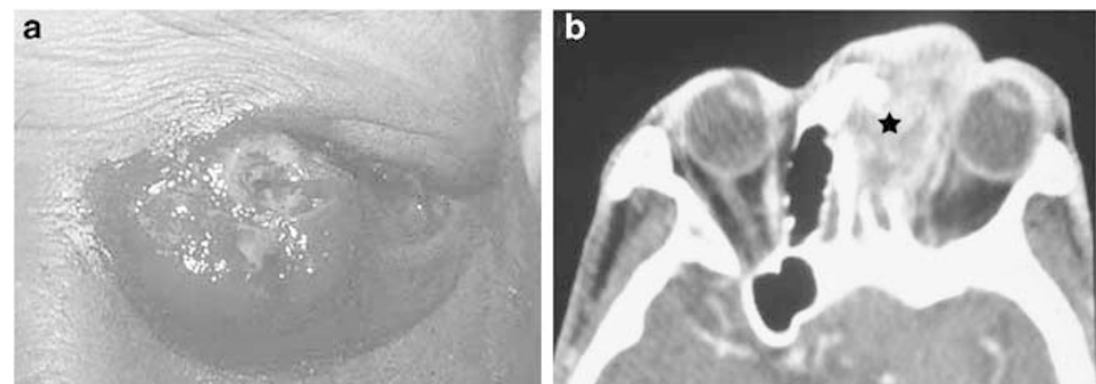

Figure 2 (a, b) A 68-year-old male (Case 9) presented with a huge left eyelid mass (a) for more than 1 year. CT scan showed a huge mass (b, asterisk) occupying the left nasal cavity and orbital cavity. 
originate from the upper nasal cavity and invade the medial orbital wall (Figure $2 b$ ). The left eye was compressed and deviated laterally. A biopsy of the left eyelid mass was performed and it revealed NPC. The patient then received radiotherapy. Nevertheless, he died 6 months later.

\section{Case 13}

This 43-year-old female patient was diagnosed with undifferentiated NPC at another hospital 2 years prior to her presentation at our clinic, with initial symptoms of left mandibular and temporal pain. She came to our hospital for help and received radiotherapy with the diagnosis of NPC at the T4N0M0 stage. She developed left trigeminal pain and limitation of extraocular movement 1 year after the radiotherapy. Ophthalmic examination revealed limited extraocular movement of all directions in the left eye. Vision was 6/6 in both eyes. An MRI study showed recurrence of NPC over the left cavernous sinus with intracranial extension. Concurrent chemotherapy and radiotherapy were given and the patient seemed to be in stable condition thereafter. After 1 year, a CT scan revealed enlargement of the pterygopalatine fossa and inferior orbital fissure (Figure 3a). Tumour recurrence with multiple cranial neuropathies was also noted 5 months later. Ophthalmic examination then revealed left eye proptosis with lagophthalmos and total ophthalmoplegia. Vision was 6/ 6.7 in the right eye with no light perception in the left eye. The pupil was dilated and fixed in the left eye. Fundus examination showed venous tortuosity and congestion in the left eye. A CT scan showed a heterogenous enhancing mass lesion, about $3 \mathrm{~cm}$ in diameter, over the left anterior inferior temporal fossa, left orbit, and lower temporal lobe (Figure 3b). The patient continued to receive chemotherapy. However, the tumour progressed to a large soft-tissue mass, about $7 \mathrm{~cm}$ in size, in the left infratemporal fossa, nasopharynx, orbit, and skull base. In spite of chemotherapy with a regimen of cisplatin, ifosfamide, and mitomycin $C$, the patient died in the 52nd month of follow-up.

\section{Discussion}

NPC is one of the most common cancers in Taiwan. It ranks as the sixth prevalent cause of malignant tumours in male Taiwanese subjects. ${ }^{6}$ NPC with orbital invasion, however, is rarely reported in the literature, and is found in a relatively low percentage of patients with orbital tumour. $^{7-11}$ In this series, 13 cases were encountered, which represented $3.2 \%$ of all orbital tumours (primary, invasive, and metastatic tumours) in patients in the study period. This percentage is high compared with the percentages in other published reports, which ranged from 0 to $3 \% .^{7-11}$ The higher percentage is probably due to the high prevalence rate of NPC in southern Chinese regions, with 15-30 per 100000 males being diagnosed with the condition per year. ${ }^{1}$

In Taiwan, NPC patients have usually presented with neck masses, nasal bleeding, and nasal congestion as the initial manifestations of the disease. ${ }^{2}$ Ocular and/or orbital manifestations of NPC include ocular motility problems (cranial nerve involvement), blurred vision, proptosis, orbital pain, and optic disc oedema. In our study, most cases presented with nasal and/or aural symptoms prior to ocular or orbital manifestations. Nine of our patients proved to be NPC victims before the tumour was found to have invaded the orbit. The period from initial NPC diagnosis to orbital invasion varied from 3 to 5 years. The other four cases in our study presented ocular and/or orbital symptoms as the initial
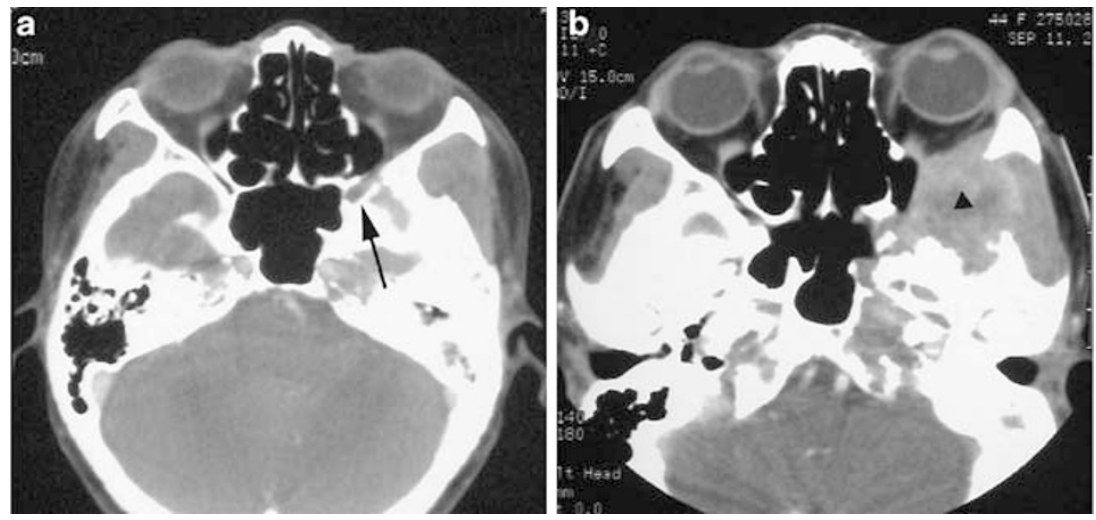

Figure 3 A 43-year-old female patient (Case 13) was diagnosed to have undifferentiated NPC. (a) CT scan revealed enlargement of the pterygopalatine fossa and inferior orbital fissure (arrow). (b, 5 months later). CT scan showed a heterogenous enhancing mass lesion (triangle), about $3 \mathrm{~cm}$ in diameter, over the left anterior inferior temporal fossa, left orbit, and lower temporal lobe. 
manifestation of NPC. One patient presented with eyelid tumour and the remaining three patients presented with proptosis. Of these four patients, one was relatively young (32-year-old) compared with the average age of NPC onset. The remaining three cases were within the average age range. Although they suffered from orbital invasion as the initial manifestation, their vision was relatively good except in one patient (Case 9). This patient's vision was severely impaired. In the other nine patients, severe visual impairment occurred in three patients (Cases $4,8,13$ ), which was mostly due to tumour compression of the optic nerve. This type of optic atrophy usually develops in the late stages of the disease and results in severe visual impairment.

In all, $22 \%$ of NPC patients were found to have cranial nerve involvement at the first examination in Taiwan. ${ }^{2}$ Patients with cranial nerve involvement may show ocular and/or orbital symptoms, such as diplopia, proptosis, and limitation of extraocular movement. Again, though, direct invasion of the orbit is rare in NPC. ${ }^{12}$ NPC is a highly infiltrative tumour and, when it does invade the orbit, it may do so via several routes. The pterygopalatine fossa and inferior orbital fissure are the most common routes of invasion, followed by invasion via the paranasal sinuses. The inferior orbital fissure represents a direct communication between the orbit and infratemporal fossa. Its most posterior part also meets the most superior extension of the pterygopalatine fossa, thus forming direct communication between the pterygopalatine fossa and the apex of the orbit. NPC involving the pterygopalatine fossa and infratemporal fossa may thus infiltrate directly into the orbit through the inferior orbital fissure. ${ }^{12}$ On the other hand, tumours in the ethmoid and/or sphenoid sinuses may erode the lamina papyracia to reach the medial orbit and retrobulbar region. The route from ethmoid/sphenoid sinuses was the second most common pathway of orbital invasion. ${ }^{12}$ On rare occasions, NPC involving the maxillary sinus may invade the inferior orbit via the floor of the orbit. As the lamina papyracia and the orbital floor are thin, they are relatively weak barriers for protecting against tumour infiltration.

Radiotherapy is the treatment of choice for NPC and its regional nodal metastasis. ${ }^{1}$ Chemotherapy was used to supplement radiotherapy for advanced nodal metastasis as well as to treat visceral metastases. ${ }^{1}$ Surgery plays a minor role in the treatment of NPC. Sometimes, however, those radioresistant nodes need to be removed by radical neck dissection. Diagnostic biopsy of the tumour is usually performed via fibreoptic scopes. This type of surgical biopsy or excision of the orbital tumour was performed in five patients in this study. The purpose of surgical intervention in the orbit was primarily for diagnosis. Radiotherapy is still the mainstay of treatment in NPC patients with orbital invasion.

In this series, nine cases were followed more than 1 year. Among a total of 13 patients, the 5-year survival rate was $28 \%$. The results of treatment in this series were fair to good and it is encouraging that radiotherapy was effective even in NPC patients with orbital invasion. In the previous literature, Heng et $a l^{13}$ reported 18 cases of orbital involvement in a Singapore study of 677 nondisseminated NPC patients. The 5-year survival rate of stage IVA (T4, N0, N1, N2, M0; T4 is tumour with intracranial extension and/or involvement of cranial nerves, infratemporal fossa, hypopharynx, or orbit) was $35 \%$ in that study. Orbital involvement confers a particularly bad prognosis in their analysis. ${ }^{13}$ Au et al. ${ }^{14}$ described a 5-year survival rate of $30 \%$ in stage IV patients in a retrospective review of 1294 consecutive nonmetastatic NPC patients. ${ }^{14}$ They found that for stage T4 NPC, intracranial extension/cranial nerve palsy and orbital involvement carried a more sinister prognosis relative to those with infratemporal fossa or hypopharynx involvement alone ${ }^{14}$ Thus, with our 5-year survival rate of $28 \%$, the results of the current study confirm that orbital involvement confers a bad prognosis in NPC patients.

\section{References}

1 Chew CT. Nasopharynx (the postnasal space). In: Kerr AG, MacKay IS, Bull TR (eds). Scott-Brown's Otolaryngology, 5th ed. Butterworths: London, 1987.

2 Hsu MM, Tu SM. Nasopharyngeal carcinoma in Taiwan, clinical manifestation and results of therapy. Cancer 1983; 52: 362-368.

3 Chong VFH, Fan YF. Skull base erosion in nasopharyngeal carcinoma: detection by CT and MRI. Clin Radiol 1996; 51: 625-631.

4 Chong VFH, Fan YF, Khoo BK. Nasopharyngeal carcinoma with intracranial spead: CT and MRI characteristics. J Comput Assist Tomogr 1996; 20: 563-569.

5 Sham JS, Cheung YK, Choy D, Chan FL, Leong L. Nasopharyngeal carcinoma: CT evaluation of patterns of tumor spread. Am J Neuroradiol 1991; 12: 265-270.

6 Cancer Registry Annual Report. 1st ed. Taipei: Department of Health, The Executive Yuan, ROC. 1997, p 6.

7 Ferry AP, Font RL. Carcinoma metastatic to the eye and orbit: a clinicopathologic study of 227 cases. Arch Ophthalmol 1974; 92: 276-286.

8 Henderson JW, Fayrow GM. Orbital Tumors, 2nd ed. Brian C. Decker, Inc.: New York, 1980; 425-450.

9 Kao LY, Ma L, Chen TT. Orbital tumor-a study of 84 cases. Afro-Asian J Ophthalmol 1985; 4: 139-144.

10 Hsu WM. Orbital tumors - a review of 200 cases. Trans Ophthalmol Soc ROC 1986; 25: 550-557.

11 Shields AJ.. Diagnosis and Management of Orbital Tumors. WB Saunders Co.: Philadelphia, 1989, pp 367-368.

12 Luo CB, Teng MMH, Chen SS, Liring JF, Guo WY, Chang T. Orbital invasion in nasopharyngeal carcinoma: evaluation 
with computed tomography and magnetic resonance imaging. Chin Med J 1998; 61: 382-388.

13 Heng DMK, Wee J, Fong KW, Lian LG, Sethi VK, Chua ET et al. Prognostic factors in 677 patients in Singapore with nondisseminated nasopharyngeal carcinoma. Cancer 1999; 15: 1912-1920.
14 Au JSK, Law CK, Foo W, Lau WH. In-depth evaluation of the AJCC/UICC 1997 staging system of nasopharyngeal carcinoma: prognostic homogeneity and proposed refinement. Int J Radiation Oncology Biol Phys 2003; 56: $413-426$. 